\title{
Rotating Wolf-Rayet stars in a post RSG/LBV phase
}

\section{An evolutionary channel towards long-duration GRBs?`}

\author{
G. Gräfener ${ }^{1}$, J. S. Vink ${ }^{1}$, T. J. Harries ${ }^{2}$, and N. Langer ${ }^{3}$ \\ 1 Armagh Observatory, College Hill, Armagh BT61 9DG, UK \\ 2 School of Physics and Astronomy, University of Exeter, Stocker Rd, Exeter EX4 4QL, UK \\ 3 Argelander-Institut für Astronomie der Universität Bonn, Auf dem Hügel 71, 53121 Bonn, Germany
}

Received 16 December 2011 / Accepted 3 October 2012

\section{ABSTRACT}

Context. Wolf-Rayet (WR) stars with fast rotating cores are thought to be the direct progenitors of long-duration gamma-ray bursts (LGRBs). A well accepted evolutionary channel towards LGRBs is chemically-homogeneous evolution at low metallicities, which completely avoids a red supergiant (RSG), or luminous blue variable (LBV) phase. On the other hand, strong absorption features with velocities of several hundred $\mathrm{km} \mathrm{s}^{-1}$ have been found in some LGRB afterglow spectra (GRB 020813 and GRB 021004), which have been attributed to dense circumstellar (CS) material that has been ejected in a previous RSG or LBV phase, and is interacting with a fast WR-type stellar wind.

Aims. Here we investigate the properties of Galactic WR stars and their environment to identify similar evolutionary channels that may lead to the formation of LGRBs.

Methods. We compile available information on the spectropolarimetric properties of 29 WR stars, the presence of CS ejecta for 172 WR stars, and the CS velocities in the environment of 34 WR stars in the Galaxy. We use linear line-depolarization as an indicator of rotation, nebular morphology as an indicator of stellar ejecta, and velocity patterns in UV absorption features as an indicator of increased velocities in the CS environment.

Results. Based on previous nebular classifications, we determine an incidence rate of $\sim 23 \%$ of WR stars with "possible ejecta nebulae" in the Galaxy. We find that this group of objects dominates the population of WR stars with spectropolarimetric signatures of rotation, while WR stars without such nebulae only rarely show indications of rotation. This confirms the correlation between rotation and CS ejecta from our previous work. The corresponding objects are most likely in an early stage after a preceding RSG or LBV phase, and have not yet lost their angular momenta due to the strong mass-loss in the WR phase. From their photometric periods we estimate rotation parameters in the range $\omega=v_{\text {rot }} / v_{\text {crit }}=0.04 \ldots 0.25$, corresponding to moderate rotation speeds of $36 \ldots 120 \mathrm{~km} \mathrm{~s}^{-1}$. These values are very uncertain, but comply with the specific surface angular momentum requirement for LGRB progenitors. From UV absorption profiles we only find weak evidence for a correlation between rotation and increased CS velocities. In particular, the CS velocities of Galactic WR stars are much lower than what is observed for GRB 020813 and GRB 021004.

Conclusions. Our results indicate that, in the Galaxy, "young" WR stars shortly after a RSG/LBV phase, show spectropolarimetric signatures of rotation. Their rotation rates are likely to be enhanced with respect to the majority of Galactic WR stars. According to their estimated specific surface angular momenta, a subgroup of stars exploding in this phase may represent an evolutionary channel towards LGRBs at high metallicities, comparable to the Galaxy. Although the UV absorption features in our sample turn out to be different from those observed in GRB 020813 and GRB 021004, it is interesting that for three WR stars with signatures of rotation, UV absorptions have previously been attributed to extended CS structures. The large size of these structures $(r \sim 100 \mathrm{pc})$ can account for the observed stability of the absorbing material in LGRB afterglows against ionizing radiation from the GRB itself. This may resolve a fundamental problem with the interpretation of the afterglow features as CS material.

Key words. stars: Wolf-Rayet - stars: rotation - stars: winds, outflows - stars: evolution - Gamma-ray burst: general - polarization

\section{Introduction}

We investigate the rotational properties of Galactic Wolf-Rayet (WR) stars, and their circumstellar (CS) environment. The main motivation is the search for observational signatures that may help to identify the progenitors of long-duration gamma-ray bursts (LGRBs). According to the collapsar model (Woosley 1993), WR stars with fast rotating cores are predicted as direct progenitors of LGRBs (cf. Sect. 1.1). Here we use the spectropolarimetric line de-polarization effect, or "line effect" as an indicator of surface rotation rates that are enhanced with respect to the majority of Galactic WR stars (cf. Sect. 1.2). Furthermore, the CS medium around WR stars can provide information about

$\star$ Tables 4 and 5 are available in electronic form at http://www. aanda.org previous evolutionary phases with dense outflows. For example, blue-shifted absorption features in some LGRB afterglows have been discussed as being due to CS ejecta from a previous red supergiant (RSG), or luminous blue variable (LBV) phase (Sect. 1.3). A comparison with the CS properties of Galactic WR stars may thus give important hints on the nature of these enigmatic explosions (Sect. 1.4).

\subsection{The collapsar model}

In his collapsar model, Woosley (1993) predicted WR stars with fast rotating cores were direct progenitors of LGRBs (MacFadyen \& Woosley 1999). In this scenario, a rotating stellar core forms a jet during its final collapse, which can only escape if the H-rich stellar envelope has been removed in earlier 
evolutionary phases. Empirically, the collapsar model has been confirmed by the association of LGRBs with Type Ib/c supernovae in several cases, such as GRB 980425 and SN 1998bw (Galama et al. 1998), and GRB 031203 and SN 2003lw (Hjorth et al. 2003; Malesani et al. 2004). Furthermore, the existing observations of LGRB afterglows are consistent with the assumption that all LGRBs contain light from an associated Type Ib/c supernova (Zeh et al. 2004), which are believed to originate from exploding Wolf-Rayet stars (Woosley et al. 1993). The association of WR stars with LGRBs thus appears to be convincing.

The question of how WR stars with large enough core angular momenta can be formed is however still under debate. The main problem is that the strong mass-loss of WR stars efficiently removes angular momentum, and thus inhibits the formation of LGRBs. Current scenarios for LGRB formation include chemically homogeneous evolution (Yoon \& Langer 2005) combined with reduced WR mass-loss rates at low metallicities (Vink \& de Koter 2005; Gräfener \& Hamann 2008), or angular momentum transfer in binary systems (e.g., Cantiello et al. 2007). An empirical confirmation of the existence of fast rotating WR stars is currently lacking. The main problem is that the spectroscopic measurement of the rotational velocities of WR stars is hindered by their fast, optically thick stellar winds (but see the example of WR 2 in Hamann et al. 2006).

\subsection{The line effect as an indicator of enhanced rotation}

An alternative method to search for rotating WR stars is via global wind anisotropies, as they are expected to be induced by rotation. In WR spectra, such anisotropies are expected to cause a line de-polarization effect, or "line effect". The reason is that WR emission lines are formed in the stellar wind, i.e. far away from the star, while the continuum radiation originates from layers much closer to the stellar surface. Continuum radiation may thus be subject to scattering in the asymmetric wind, and show a stronger linear polarization than the lines. In single stars the interpretation of the line effect is unambiguous - the detection of a line effect means that the electrons in the wind are aspherically distributed. However a non-detection may arise if a nonspherical wind is viewed at an inopportune orientation (a face-on disc for example).

The line effect, originally observed in classical Be stars (Poeckert \& Marlborough 1977), has since been discovered in LBVs (e.g., Schulte-Ladbeck et al. 1994), Wolf-Rayet stars in both the Milky way (e.g., Harries et al. 1998) and the LMC (Vink 2007) and O stars (Harries \& Howarth 1996; Vink et al. 2009). In the present work we use the results by Harries et al. (1998) as an indicator for rotation of Galactic WR stars. The properties of the six stars in this sample with positive detections of a line effect, and their interpretation as rotating WR stars, have recently been discussed by Vink et al. (2011a).

Generally, linear polarization may also arise from intrabinary scattering, leading to a phase-locked variation of the polarization magnitude and direction with orbital phase (Brown et al. 1978). Such polarization signatures have been observed in close $\mathrm{WR}+\mathrm{O}$ and $\mathrm{O}+\mathrm{O}$ star binaries e.g. V444 Cyg (St.-Louis et al. 1993), CQ Cep (Harries \& Hilditch 1997), LZ Cep (Berdyugin \& Harries 1999). The binary separation must necessarily be small, since a significant $>1 \%$ fraction of the brighter star's flux must be scattered off its companion's wind; a constraint which implies binary periods of days rather than years. Such short-period binaries are easily detected via photometric and/or radial velocity variation, and thus a continuum polarization signature from a star without evidence for short-period binarity can be readily attributed to asymmetries in the stellar wind alone.

A plausible explanation for the appearance of a line-effect is the presence of a substantial wind asymmetry associated with rapid rotation (cf. the classical Be star case). For WR stars an equator-to-pole density contrast of a factor of 2-3 results in a measurable line-effect (cf., Harries et al. 1998). An estimation of the associated rotation rates is difficult, as the structure of rotating radiatively driven winds is still a matter of research. Predictions include, e.g., wind compressed disks (Bjorkman \& Cassinelli 1993), bipolar outflows due to gravity darkening at the equator (Owocki \& Gayley 1997), or equatorially enhanced outflows due to the bi-stability mechanism (Pelupessy et al. 2000). For WR winds, the effect of rotation has not yet been investigated. Their strong $\Gamma$-dependence (Gräfener \& Hamann 2008; Vink et al. 2011b) however implies that rotation may cause enhanced mass loss at the equator, where the effective gravity is lower (cf. the discussion in Gräfener et al. 2011). Observationally, Harries et al. (1999) find strong evidence that the wind of WR 6 is azimuthally structured. Due to the multitude of potentially important physical effects it is presently difficult to obtain quantitatively reliable estimates of the influence of rotation on the line effect in WR stars.

Based on their wind-compressed disk models, Ignace et al. (1996) found that WR stars may show significant wind asymmetries for rotational velocities above $\sim 16 \%$ of the break-up speed, i.e. above a distinct rotation parameter $\omega=v_{\text {rot }} / v_{\text {crit }} 1$. They propose that the line-effect stars are those whose rotational velocities exceed this (very uncertain) limit, i.e., that these stars represent the high-velocity tail of the rotational velocity distribution. This is in accordance with the results from Harries et al. (1998), who found that their polarization measurements are in agreement with a minority of $\sim 20 \%$ of asymmetric WR winds observed at arbitrary viewing angles, while the majority of WR stars has no significant deviations from radial symmetry. We thus conclude that the connection between line effect and rotation is plausible, and that the line effect stars most likely represent a minority $(\sim 20 \%)$ of WR stars with larger rotation parameters $\omega$ than the majority of WR stars, i.e. their rotation parameters are enhanced. In the following we classify this group of objects as WR stars with enhanced rotation.

Alternative scenarios for the occurrence of a line effect are wind asymmetries due to global magnetic fields, or the presence of co-rotating interaction regions (CIRs) in WR-type winds (cf. Cranmer \& Owocki 1996). As we will discuss in Sect. 3.1.2, magnetic fields are unlikely to be the reason for the line effect in WR stars because the required field strengths would be extremely high. CIRs, on the other hand, are commonly observed via line-profile variations (LPVs) in WR spectra (Morel et al. 1997, 1999; Flores et al. 2007; Chené \& St.-Louis 2010), in agreement with theoretical models by Dessart \& Owocki (2002). Whether CIRs are able to cause a global wind asymmetry that is sufficient to cause a line effect, has not yet been investigated. However, even in this case the line effect would be indicative of stellar rotation.

As discussed above, it is a plausible assumption that the observed line effect for WR stars is a signature of enhanced rotation. In the following we use this assumption to investigate the rotational properties of the Galactic WR sample. Notably, we expect that the associated rotational velocities lie significantly below the critical speed.

1 For the definition of the critical, or break-up velocity $v_{\text {crit }}$ see Sect. 3.1.2. 


\subsection{Circumstellar absorption features in LGRB afterglow spectra}

Circumstellar absorption features in LGRB afterglow spectra have been discussed as a possible route to identification of the progenitors of LGRBs. Many LGRB afterglows show absorption features with complex velocity patterns, that partly originate from high ionization stages such as $\mathrm{C}$ Iv, $\mathrm{Nv}$, O vi, Si Iv (e.g., Fox et al. 2008). A subgroup of LGRB afterglows has been found to exhibit blue-shifted absorptions with velocities typical for WR-type stellar winds (3000-4000 $\mathrm{km} \mathrm{s}^{-1}$ ), in combination with strong absorption features with velocities of $130-630 \mathrm{~km} \mathrm{~s}^{-1}$ in $\mathrm{C}$ IV and Si IV which are broadened, and display a multi-component structure (GRB 020813 and GRB 021004; Schaefer et al. 2003; Mirabal et al. 2003; Fiore et al. 2005; Starling et al. 2005). van Marle et al. (2005) used hydrodynamical simulations to suggest that these features may arise when a CS ejecta shell from a previous RSG/LBV phase breaks up due to the interaction with the fast WR wind. In order to prevent the CS material from being completely ionized by the GRB this model requires additional conditions, such as a highly clumped CS medium (Schaefer et al. 2003), or a structured jet (Starling et al. 2005).

Fox et al. (2008) investigated high-ionization features in the spectra of seven early GRB afterglows (including the CS ejecta candidate GRB 021004) and found high-velocity C IV features $\left(500-5000 \mathrm{~km} \mathrm{~s}^{-1}\right)$ consistent with a WR-type wind in six cases. Furthermore they identified strong absorption components near zero velocity, blue-shifted line wings with velocities up to $100-150 \mathrm{~km} \mathrm{~s}^{-1}$, and weak multi-component profiles with velocities up to several hundred $\mathrm{km} \mathrm{s}^{-1}$, from which they concluded that they are all likely of interstellar origin. In particular, they interpreted the weak C IV and Si Iv multi-component features with velocities of several hundred $\mathrm{km} \mathrm{s}^{-1}$ as being due to galaxy outflows, in analogy to absorption features commonly observed in high-redshift damped Lyman $\alpha$ galaxies.

As the much stronger multi-component features in GRB 021004 were not included in their analysis, it is however not clear whether these would fall into the same category or not. One goal of our present work is to investigate the Galactic WR sample for the presence of similar absorption features as in GRB 020813 and GRB 021004.

\subsection{Ejecta nebulae around Galactic WR stars, and stellar rotation}

The presence of ejecta nebulae around WR stars is indicative of a previous episode of strong mass loss with small outflow velocities, such as the RSG, or LBV phase. Within a single star scenario, many WR stars are thought to have lost their hydrogen-rich envelopes in these phases (e.g., Langer et al. 1994; Meynet \& Maeder 2005). According to the calculations of van Marle et al. (2005), the remaining dense shells are swept up by the much faster WR wind, until they break up and dissipate $\sim 80000 \mathrm{yr}$ after the beginning of the WR phase. During this breakup phase, the shell material can reach velocities of $150-700 \mathrm{~km} \mathrm{~s}^{-1}$, with a high velocity dispersion. The resulting CS absorptions are expected to show up in the form of strong blue-shifted broadened/multi-component absorption features, as described in Sect. 1.3. The presence of CS shells with such highvelocity features may thus be used to identify WR stars in a relatively early stage after the RSG/LBV phase. Such "young" WR stars are likely to show enhanced rotation rates, as they have only recently developed the strong WR star wind. WR stars exploding in this phase are thus promising candidate LGRB progenitors (Petrovic et al. 2005).

The main goal of our present work is to investigate whether there exists a correlation between CS, and rotational properties for Galactic WR stars, which would support the above scenario. Connections between CS, and intrinsic stellar properties of WR stars have been discussed earlier. Nichols \& Fesen (1994) measured CS velocity displacements from IUE high-resolution data, and identified CS high-velocity features for three stars that have later been found to show a line effect (WR 6, WR 40, \& WR 136; cf. Harries et al. 1998). Stock \& Barlow (2010) compiled a list of ejecta nebulae around Galactic WR stars. Based on the spectropolarimetric detections by Harries et al. (1998), Vink et al. (2011a) highlighted a correlation between the presence of ejecta nebulae and a line effect, and discussed the relevance of linear spectropolarimetry in the identification of LGRB progenitors.

Here we compile information about the spectropolarimetric, and CS properties of Galactic WR stars from various surveys, including their CS velocities. In this way we can define criteria for the presence of "possible ejecta nebulae" from a previous RSG/LBV phase, and investigate correlations with (non)detections of a line effect, and enhanced velocity dispersions within the CS material. Based on this data we wish to identify WR stars with enhanced rotation rates (and thus potential LGRB progenitors) in the Galaxy, and the evolutionary channels that lead into these stages. The latter is of general interest for the question how LGRBs can form, but may also set constraints on the complex physics of stellar evolution with rotation.

\section{The Galactic Wolf-Rayet sample}

In this section we compile information about the spectropolarimetric, and CS properties of Galactic WR stars. We constrain the rotational properties by the detection of a line effect in linear spectropolarimetry (Sect. 2.1), and the evolutionary stage by the presence or absence of ejecta or ring nebulae (Sect.2.2). In addition, we examine IUE high-resolution spectra for CS absorption features with velocity patterns similar to GRB 020813 and GRB 021004 in Sect. 2.3. Correlations between these characteristics are investigated in Sect. 2.4.

\subsection{Spectropolarimetric properties of Galactic WR stars}

The spectropolarimetric properties of a sample of 29 Galactic WR stars was compiled by Harries et al. (1998), who found 9 stars with a line effect in their sample. Four of these stars are known binaries, and Harries et al. identified phaselocked modulations of the line effect in 3 of them, i.e., their polarization is due to binarity. For the remaining 6 stars with a line effect, the polarization is thus likely due to intrinsic properties of the WR star itself, such as wind anisotropies due to rotation (cf. Sect. 1.2). One of these 6 objects (WR 136) is only detected by Whitney et al. (1988), but not by Schmidt (1988); Schulte-Ladbeck (1994); Harries et al. (1998); for a more detailed discussion see Vink et al. (2011a). In Table 1 we have compiled the spectropolarimetric properties of the 29 sample stars, together with CS properties from Sects. 2.2, and 2.3.

\subsection{Ring nebulae around Galactic WR stars}

To constrain the CS properties of the stars in Table 1, we use the results of the Southern survey of ring nebulae around Galactic 
Table 1. WR stars with spectropolarimetry from Harries et al. (1998): circumstellar, and spectropolarimetric properties of the sample stars.

\begin{tabular}{lccccc}
\hline \hline WR & Spec. type & LE & RN & NF & C IV \\
\hline 006 & WN4b & + & W, E & NF++ & 84.6 \\
016 & WN8h & + & W/E & NF- & 278.8 \\
040 & WN8h & + & W/E & NF++ & 287.1 \\
134 & WN6b & + & W & NF+ & 113.3 \\
136 & WN6 & + & W/E & NF+ & 145.2 \\
137 & WC7+OB & + & - & NF+ & 110.8 \\
\hline 014 & WC7 & - & R & NF- & 67.4 \\
018 & WN4b & - & W, W/E & & \\
024 & WN6ha & - & - & NF+ & 116.7 \\
078 & WN7h & - & - & NF- & 73.2 \\
104 & WC9 & - & - & & \\
110 & WN5-6b & - & - & & \\
111 & WC5 & - & - & NF- & 91.4 \\
121 & WC9 & - & - & & \\
127 & WN3b+O9.5V & - & - & & \\
128 & WN4(h) & - & R & NF- & 59.5 \\
133 & WN5o+O9I & - & RN?? & NF+ & 109.9 \\
135 & WC8 & - & - & NF+ & 98.3 \\
138 & WN5o+B? & - & - & NF+ & 98.4 \\
139 & WN50+O6 & $*$ & - & NF- & 62.8 \\
141 & WN6+O5 & $*$ & - & & \\
143 & WC4 & - & - & & \\
148 & WN8h & - & - & NF++ & - \\
150 & WC5 & - & - & & \\
152 & WN3(h) & - & - & & \\
$153 a b$ & WN6o/CE+O6I & - & RN?? & NF- & 86.8 \\
155 & WN6o+O9II/Ib & $*$ & - & NF- & 116.6 \\
156 & WN8h & - & - & & \\
157 & WN5o(+B1II) & - & - & & \\
\hline \multicolumn{7}{c}{} & & & & & \\
\hline
\end{tabular}

Notes. WR designations by van der Hucht (2001); Spectral types from Harries et al. (1998); LE: line effect according to Harries et al. (1998) (+ line effect, * line effect due to binarity, - non-detection); RN: detections of ring nebulae, with classifications from Table 5; NF: detections of CS velocity displacements according to Nichols \& Fesen (1994) (++ safe detection, + detection with possible super-shell, non-detection); $\mathrm{C}_{\text {Iv }}$ : CS broadening velocities (FWHM in $\mathrm{km} \mathrm{s}^{-1}$ ) from high-ionization features (average of $\mathrm{C}_{\text {Iv }}$ 1548, 1550 from Table 4).

WR stars by Marston et al. (1994a,b); Marston (1997), and of the Northern survey by Miller \& Chu (1993). In Table 5 we have compiled all objects that were included in these surveys (please note the changes in some stellar designations with respect to the original works). The total sample in Table 5 comprises 172 WR stars from the catalogue of van der Hucht (2001). The missing 54 stars from this catalogue are mostly optically very faint, such as stars from the Galactic center region, or stars that have been added only recently to the catalogue. Here we use the 172 stars from Table 5 as an unbiased Galactic WR sample.

The classifications given in Table 5 are based on the three categories of ring nebulae introduced by Chu (1981): radiatively excited $\mathrm{H}_{\text {II }}$ regions (R), wind-blown bubbles (W), and stellar ejecta (E). As Miller \& Chu (1993) only distinguished between different detection probabilities, i.e. ring nebulae (RN), probable ring nebulae (RN?), and possible ring nebulae (RN??), we have added additional (re)classifications from Chu (1991); Stock \& Barlow (2010) in Table 5.

The classification scheme by Chu (1981) is based on the nebula morphology in different bandwidths. The categories R, W, and $\mathrm{E}$ are thus chiefly morphological indicators, and the physical interpretation implied by the designations $\mathrm{R}, \mathrm{W}$, and $\mathrm{E}$ may not necessarily reflect the actual physical state of the system.
Indeed, nebulae of categories $\mathrm{W}$ and $\mathrm{E}$ have been confirmed to contain nucleosynthetic products (see Table $1 \&$ the discussion in Sect. 2 in Stock \& Barlow 2010). Ring nebulae of types W, E, as well as mixed types containing these components, thus likely contain stellar ejecta from previous RSG, or LBV phases. In the following we use this physical interpretation, and denote such nebulae as "possible ejecta nebulae" of types W, E.

Based on 172 stars in total, we find 54 stars (31\%) with ring nebulae, of which $40(23 \%)$ have been classified as types W, E. Of the remaining 14 stars, $10(6 \%)$ have been classified as $\mathrm{R}$, and $4(2 \%)$ are unclassified. We thus have $40+4=44$ stars $(26 \%)$ in our sample that may have ejecta nebulae of types W, E. These numbers agree well with the numbers given by Marston (1997).

From the sample of 29 WR stars with spectropolarimetric measurements in Table 1, 10 (34\%) have positive detections of ring nebulae, well in agreement with the Galactic average obtained above. 6 of these $(21 \%)$ have classifications of types W, E. 2 stars $(7 \%)$ are classified as R, and another 2 are not classified. Also these numbers reflect the Galactic average, so that the sample in Table 1 can be seen as representative. We note that the two unclassified cases are "possible" detections (RN??) by Miller \& Chu (1993), i.e., they are very uncertain.

The striking fact that 5 of the 6 stars with a line effect in Table 1 have possible ejecta nebulae, has recently been discussed by Vink et al. (2011a). We will discuss the statistics of the complete sample from Table 1 in Sect. 2.4.

\subsection{Circumstellar velocity patterns}

In this section we investigate how the UV absorption features of Galactic WR stars compare with the putatively CS absorptions in GRB 020813 and GRB 021004. As with the GRB afterglows, the WR absorption features will include a mixture of CS and interstellar components, i.e. their interpretation is often ambiguous. For this reason we will use the results from the present section in Sect. 2.4 to find statistical correlations between rotational and CS properties.

We start with an inspection of the C IV resonance doublet at 1548.2, 1550.8 $\AA$ for all Galactic WR stars with available IUE high-resolution data. We aim to find $\mathrm{C}$ IV absorption features similar to GRB 020813 and GRB 021004, i.e. strong broadened/multi-component absorptions with velocity displacements of several hundred $\mathrm{km} \mathrm{s}^{-1}$. We therefore measure the FWHM (full width half minimum) velocity spread of all observed C IV absorption components, including CS and interstellar features. The broadening velocities should thus not be interpreted as physical broadening velocities. We further note that we do not perform an absolute velocity calibration, it is therefore not certain that the measured velocities correspond to blueshifts. Due to the limited data quality, and the ambiguities in the identification of the absorption components, the uncertainties in our measurements are likely of the order of $10 \%$ or higher.

The results of our Civ measurements are compiled in Table 4, and for the cases overlapping with our spectropolarimetric sample average values of the two doublet components are listed in Table 1. In most cases the measured $\mathrm{C}$ IV broadening velocities turn out to be much smaller $\left(<200 \mathrm{~km} \mathrm{~s}^{-1}\right)$ than the velocities observed GRB afterglow features. Only in two cases, WR 16 and WR 40, we find broadening velocities in the range of $\sim 300 \mathrm{~km} \mathrm{~s}^{-1}$. As demonstrated in Fig. 2 for the case of WR 40, these features however display a very similar type of line-profile variability which is coupled to variations in the terminal wind 
speed (Smith et al. 1985). The broad C IV absorptions in these two objects thus likely originate from variable wind structures, rather than from CS material. This is supported by the analysis of UV absorption features of WR 40 by Smith et al. (1984), who found much lower blue-shifts up to $150 \mathrm{~km} \mathrm{~s}^{-1}$ in $\mathrm{C}$ IV.

A dedicated search for CS signatures in the available IUE high-resolution data of 35 Galactic WR stars has been performed by Nichols \& Fesen (1994); Nichols-Bohlin \& Fesen (1986, 1990, 1993). These studies also include lower ionization stages of various elements, and concentrate on velocity displacements of individual sub-components in the partly very complex UV absorption spectra (similar to previous studies by Smith et al. 1980, 1984; St.-Louis \& Smith 1991). Nichols \& Fesen (1994) identified high-velocity absorption features, "conservatively defined to be components separated from the main component by at least $45 \mathrm{~km} \mathrm{~s}^{-1}$ ", in 16 of the 35 stars. As 10 of these are associated with known super-shells of star forming regions, such as Carina OB1/OB3, Cyg OB1, and Sco OB1, Nichols \& Fesen concluded safe detections of CS high velocity components for only 4 of the 22 isolated WR stars in the sample. We have compiled their results in Table 4, and have added the information for the 18 stars overlapping with our spectropolarimetric sample from Table 1.

In many cases, our results are in agreement with Nichols \& Fesen (1994), in the sense that stars for which Nichols \& Fesen find large velocity displacements, also tend to show enhanced broadening velocities in C IV. In some cases, lines with small velocity displacements may however be significantly broadened. For example Smith et al. (1980) found no velocity displacement in $\mathrm{C}$ IV for WR 6, but we find a substantial broadening of $85 \mathrm{~km} \mathrm{~s}^{-1}$. In Fig. 1, the C IV absorption profiles of WR 6 indeed appear to be dominated by a strong interstellar component at rest wavelength. The line profiles are however substantially broadened and asymmetric, potentially due to an unresolved blue-shifted CS component. Indeed, based on the analysis of a whole range of ionization species, Nichols \& Fesen (1994) detected significant CS velocity displacements for this star. This case demonstrates that line widths are a good additional tool to identify enhanced CS velocity dispersions. In Fig. 1 we have compiled more examples of typical absorption profiles, also to visualize the tentative classifications given in Table 4.

\subsection{Correlations between circumstellar and spectropolarimetric properties}

In the sample of 29 stars with spectropolarimetric measurements from Table 1, we found 6 stars with possible ejecta nebulae of types W, E, plus 2 stars with uncertain detections and no classification (RN??). If we only concentrate on the stars with classifications, as many as 5 of 6 stars with a line effect fall into our category of stars with possible ejecta nebulae. Conversely, 5 of the 6 stars with possible ejecta nebulae show a line effect. This implies a very strong correlation between both properties. Indeed, for the null hypothesis that no correlation exists between both properties, the probability of achieving this result by chance is only $0.03 \%$. If we also include the two uncertain cases without nebular classifications as candidates for possible ejecta nebulae, this value increases to $0.3 \%$, i.e., the correlation is still significant.

The correlation of high-velocity absorption components (according to Nichols \& Fesen 1994) with the line effect, turns out to be more ambiguous. The likely reason is that the absorption features are not necessarily of CS nature. Moreover, according to van Marle et al. (2005), the detection probability

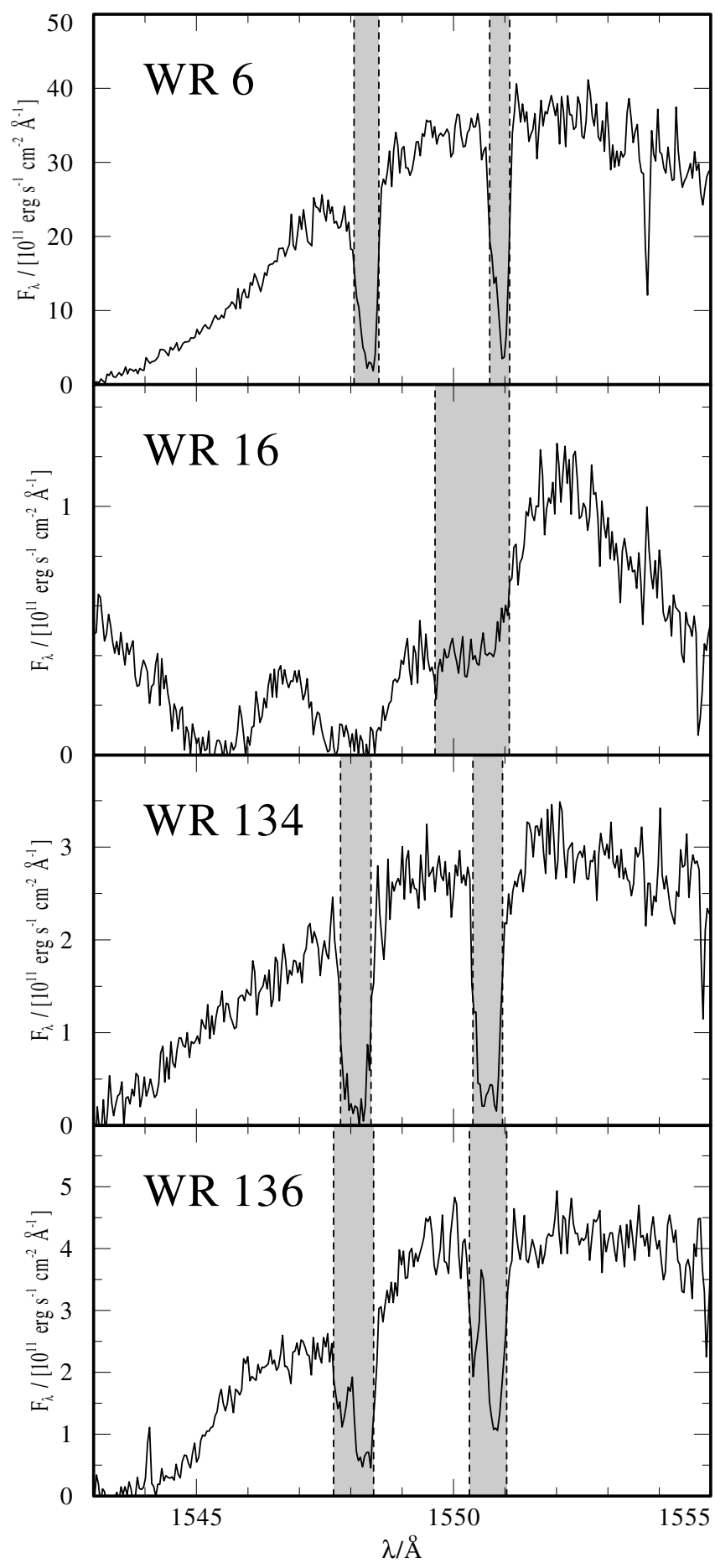

Fig. 1. Examples for absorption features in C Iv 1548, 1550 from IUE high-resolution data. The line widths given in Table 4 , are indicated as grey shaded areas. The broad feature in the spectrum of WR 16 shows a similar variability as WR 40 (cf. Fig. 2), and is thus likely of intrinsic nature.

of CS high-velocity features is limited to $20-60 \%$, due to the clumpiness of the CS material.

From 6 stars with a line effect in Table 1, 5 objects are detected by Nichols \& Fesen. In total, we find 10 positive, and 8 negative detections for the 18 objects overlapping with our spectropolarimetric sample. If there no correlation exists between both properties, the probability for this result would be $18.0 \%$. We thus find evidence for a correlation, but the evidence is weak. 


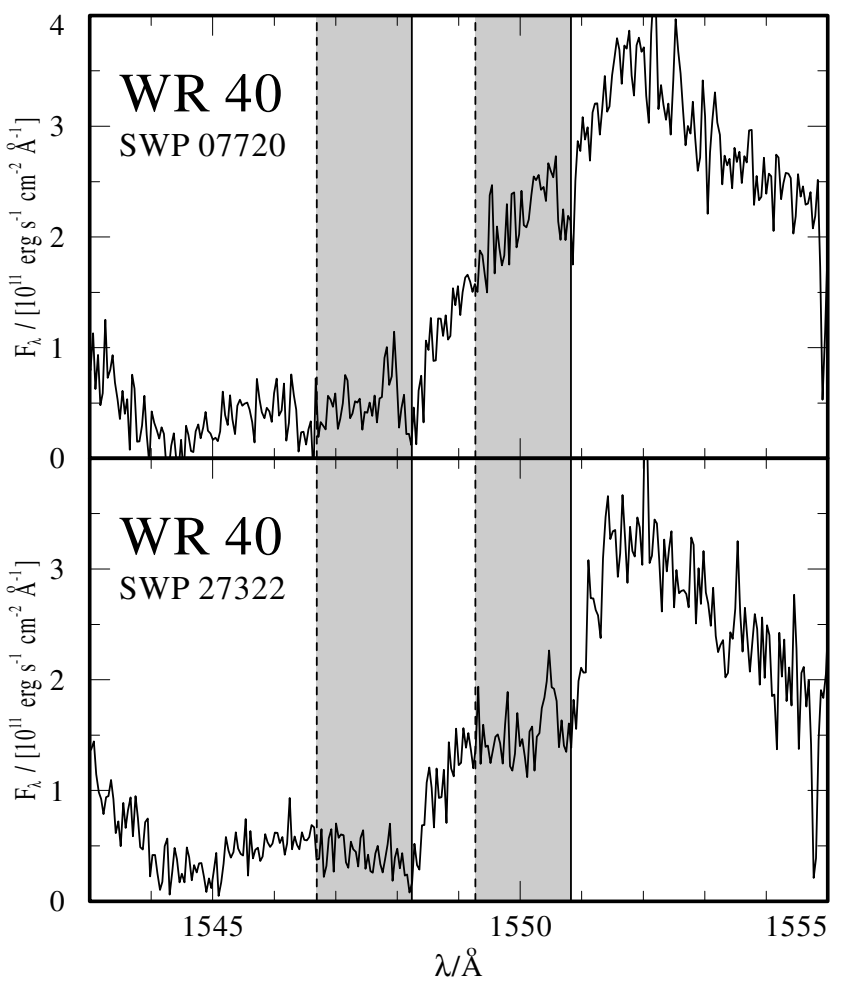

Fig. 2. Variability in the $\mathrm{C}_{\mathrm{IV}}$ absorption features of WR 40. The broad features in the spectrum from 18/12/85 (bottom panel) are absent in the spectrum from 20/01/80 (top panel). The features are thus likely not of CS nature.

When we consider the $C_{\text {IV }}$ broadening velocities in the last column of Table 1 , it is striking that 5 out of 6 cases in the line-effect sample have broadening velocities significantly larger than $100 \mathrm{~km} \mathrm{~s}^{-1}$, while this is only the case for 3 out of 11 non line-effect stars. This is also reflected in a much higher average of $170.0 \pm 89.6 \mathrm{~km} \mathrm{~s}^{-1}$ for stars with a line effect, vs. $89.2 \pm 21.0 \mathrm{~km} \mathrm{~s}^{-1}$ for stars without. From a two-distribution Kolmogorov-Smirnov test with the values in Table 1 we obtain a probability of only $7.4 \%$ that the velocity distributions for the line-effect and the non line-effect stars are the same. If we exclude WR 16 and WR 40, whose broad C IV features are likely due to intrinsic variability (cf. Sect. 2.3), this probability rises to $30 \%$, mainly because the test becomes very weak due to the low number of data points in the line-effect sample. For this case also the average broadening velocity goes down to $113.5 \pm 24.8 \mathrm{~km} \mathrm{~s}^{-1}$, which is still higher than the value for the non line-effect sample but just within the standard deviation. The overall statistical evidence for the detection of increased velocities in the UV absorption profiles (including the results from Nichols \& Fesen) thus stays weak.

We thus conclude that there is a strong correlation between the presence of possible ejecta nebulae and the detection of a line effect for the $29 \mathrm{WR}$ stars in Table 1 . However, the statistical evidence for increased velocities in the UV absorption profiles of WR stars with a line effect is weak.

\section{Discussion}

The main result of this work is that the $\sim 23 \%$ of Galactic WR stars with possible ejecta nebulae dominate the group of WR stars with enhanced rotation rates, as detected by spectropolarimetry. As we will discuss in the following, this is pertinent to the question of how rotating massive stars evolve. Moreover, similar objects have been discussed as LGRB progenitors, based on the observed UV absorption features in GRB 020813 and GRB 021004 (cf. Sect. 1.3). This raises the question whether stars in this phase may represent an evolutionary channel towards LGRBs. In Sect. 3.1 we discuss the relevance of our results for these questions. In Sect. 3.2 we discuss to which extent the absorption features in Galactic WR spectra compare to the absorption features in LGRB afterglows.

\subsection{Galactic WR stars with enhanced rotation}

In Sect. 2.4 we confirmed the strong correlation between the detection of a line effect, and the presence of CS ejecta from Vink et al. (2011a) for the complete sample of Galactic WR stars from Harries et al. (1998). This implies that in our Galaxy mostly "young" WR stars, just after a preceding RSG or LBV phase, show signatures of enhanced rotation. Apparently, these stars have been able to retain angular momentum throughout their preceding evolution, until the beginning of the WR phase. Based on the fact that 5 out of 6 WR stars with signatures of rotation have circumstellar ejecta shells, Vink et al. (2011a) concluded that WR stars ending their lives in such an early WR stage may be promising candidates for LGRB progenitors.

In this work we investigated the complete sample of 29 WR stars with spectropolarimetric measurements from Harries et al. (1998), including non-detections. Based on our criteria for "possible ejecta nebulae", we confirmed the strong correlation from Vink et al. (2011a), and additionally found a very small incidence of rotational signatures for stars without possible ejecta nebulae. This indicates that in the Galaxy, post-RSG/LBV stars tend to lose a substantial amount of their (surface) angular momentum during their subsequent evolution, most likely due to the strong mass loss in the WR stage.

The group of Galactic WR stars with possible ejecta nebulae $(\sim 23 \%)$ thus seems to dominate the group of WR stars with enhanced rotation rates, according to spectropolarimetry $(\sim 20 \%$ in the sample of Harries et al. 1998). The only object with a line effect that does not display a nebulosity, is the WC 7 component in the binary system WR 137. As previously discussed by Chené $\&$ St.-Louis $(2010)^{2}$, this more evolved object may represent an evolutionary channel towards LGRBs via chemically homogeneous evolution (Yoon \& Langer 2005), or binary interaction (Cantiello et al. 2007). However, a key ingredient for GRB formation within these scenarios is a reduction of WR mass-loss at low metallicities (cf. Vink \& de Koter 2005; Gräfener \& Hamann 2008), so that Galactic WR stars in these phases are not expected to explode as LGRBs.

In the following we will chiefly concentrate on the rotating post-RSG/LBV objects, and discuss how our findings compare with evolutionary models, and what their implications for LGRB formation are.

\subsubsection{Evolutionary status}

To investigate the evolutionary status of our line-effect stars, we have compiled stellar parameters from Hamann et al. (2006); Herald et al. (2001) for the 5 WN stars with a line effect in Table 2. For the WC component in WR 137 we adopt the parameters of the single WC 7 star WR 90 from Dessart et al. (2000), which likely resemble the parameters of WR 137. Due to the

${ }^{2}$ Chené \& St.-Louis adopt a very small mass $\left(M \sin ^{3} i=3.4 \pm 1.0 M_{\odot}\right.$ Lefèvre et al. 2005) and thus derive a somewhat larger value of $\omega=$ $v_{\text {rot }} / v_{\text {crit }} \sim 0.5$, compared to this work $(\omega \sim 0.3)$. 
Table 2. Stellar parameters for WR stars with a line effect.

\begin{tabular}{cccccccc}
\hline \hline WR & $\begin{array}{c}T_{\star} \\
{[\mathrm{kK}]}\end{array}$ & $X_{\mathrm{H}}$ & $\begin{array}{c}\log L \\
{\left[L_{\odot}\right]}\end{array}$ & $\begin{array}{c}R_{\star} \\
{\left[R_{\odot}\right]}\end{array}$ & $\begin{array}{c}M_{\star} \\
{\left[M_{\odot}\right]}\end{array}$ & $\Gamma_{\mathrm{e}}$ & $\begin{array}{c}\log \left(\dot{M} D^{\frac{1}{2}}\right) \\
{\left[M_{\odot} / \mathrm{yr}\right]}\end{array}$ \\
\hline 006 & 89.1 & 0.0 & 5.6 & 2.65 & 17.9 & 0.342 & -4.0 \\
016 & 44.7 & 0.25 & 6.15 & 19.9 & 40.1 & 0.678 & -4.0 \\
& 41.7 & 0.23 & 5.68 & 12.3 & 20.0 & 0.453 & -4.3 \\
040 & 44.7 & 0.23 & 6.05 & 17.7 & 34.3 & 0.618 & -3.8 \\
& 45.0 & 0.15 & 5.61 & 10.6 & 18.2 & 0.397 & -4.0 \\
134 & 63.1 & 0.0 & 5.6 & 5.29 & 17.9 & 0.342 & -4.1 \\
136 & 70.8 & 0.12 & 5.4 & 3.34 & 13.7 & 0.316 & -4.2 \\
\hline 090 & 71.0 & 0.0 & 5.5 & 3.71 & 15.6 & 0.311 & -4.1 \\
\hline
\end{tabular}

Notes. Stellar parameters for WN stars are from Hamann et al. (2006), plus results for WR 16, \& WR 40 from Herald et al. (2001). Because the distances towards WR 16, \& WR 40 are not known, the derived luminosities differ. For the WC 7 component in WR 137, we give parameters derived for the similar WC 7 star WR 90, from Dessart et al. (2000). Masses $M_{\star}$, and Eddington factors $\Gamma_{\mathrm{e}}$ are obtained from the massluminosity relations for He-burning stars by Gräfener et al. (2011). The spectroscopically determined mass-loss rates $\dot{M}$ are given as a function of the wind clumping parameter $D$, which is expected to lie in the range $D=4-16$ for WR stars (Hamann \& Koesterke 1998).

unknown distance towards WR 16 and WR 40 (both WN 8 subtypes), the luminosities of these two stars are unknown. Based on different luminosity vs. spectral subtype calibrations, Herald et al. (2001) determine lower luminosities for these two stars than Hamann et al. (2006). We note that the calibration for WN 8 subtypes according to Hamann et al. relies on luminous H-rich WN 8 stars in young stellar clusters with known distances. As WR 16 and WR 40 display a different spectral morphology than these objects, they likely belong to the group of classical, He-burning WR stars with lower luminosities.

Based on the lower luminosities, all WN stars in our sample are in agreement with being post-RSG objects (for WR 16 and WR 40 we can of course not exclude a post-LBV origin). They display a relatively small amount of residual surface hydrogen $\left(X_{\mathrm{H}}=0-0.25\right)$, and mostly low surface temperatures $<70 \mathrm{kK}$.

The probability to find WN stars with and without residual surface hydrogen at such low temperatures has been investigated by Hamann et al. (2006), using population synthesis models based on single star evolution models by Meynet \& Maeder (2003). Based on rotating models, a significant population of cool WR stars $\left(T_{\star}<70 \mathrm{kK}\right)$ with residual surface hydrogen is expected in the post-RSG regime (i.e. with $\log \left(L / L_{\odot}\right) \lesssim 5.9$ ). For non-rotating models, the amount of $\mathrm{H}$-rich material on top of the He-core is so small that the stars evolve extremely fast towards the He-main sequence after leaving the RSG phase, i.e., they are almost un-detectable. Within a single star scenario, rotation thus seems to be a mandatory condition to form the objects in Table 2.

\subsubsection{Rotational velocities}

An estimate of the rotational velocities at the stellar surface can be obtained from photometric variations. Photometric periods have been detected for all stars in our line-effect sample. Chené \& St.-Louis (2010) recently proposed that this periodicity could be caused by co-rotating interaction regions (CIRs) in the winds of WR stars, and determined rotational velocities for three of our sample stars. In Table 3 we have compiled our own estimates (that are basically in agreement with the ones from Chené \& St.-Louis), based on observed photometric periods
Table 3. Rotational properties inferred from photometric variability.

\begin{tabular}{ccccccccc}
\hline \hline WR & $\begin{array}{c}P \\
{[\mathrm{~d}]}\end{array}$ & $\begin{array}{c}\tau_{\text {hyd }} \\
{[\mathrm{d}]}\end{array}$ & $\begin{array}{c}v_{\text {esc }} \\
{\left[\frac{\mathrm{km}}{\mathrm{s}}\right]}\end{array}$ & $\begin{array}{c}v_{\text {crit }} \\
{\left[\frac{\mathrm{km}}{\mathrm{s}}\right]}\end{array}$ & $\begin{array}{c}v_{\text {rot }} \\
{\left[\frac{\mathrm{km}}{\mathrm{s}}\right]}\end{array}$ & $\begin{array}{c}\log (j) \\
{\left[\frac{\mathrm{cm}^{2}}{\mathrm{~s}}\right]}\end{array}$ & $\begin{array}{c}\frac{M_{\star}}{\dot{M} \sqrt{D}} \\
{\left[10^{5} \mathrm{yr}\right]}\end{array}$ & Ref. \\
\hline 006 & 3.77 & 0.02 & 1605 & 920 & 36 & 17.8 & 1.8 & $(1)$ \\
016 & 10.7 & 0.18 & 787 & 411 & 58 & 18.7 & 4.0 & $(2)$ \\
040 & 4.76 & 0.15 & 809 & 444 & 113 & 18.9 & 1.8 & $(2)$ \\
134 & 2.25 & 0.05 & 1136 & 651 & 119 & 18.6 & 2.3 & $(3)$ \\
136 & 4.55 & 0.03 & 1250 & 731 & 37 & 17.9 & 2.2 & $(4)$ \\
137 & 0.83 & 0.03 & 1266 & 743 & 226 & 18.7 & 2.0 & $(5)$ \\
\hline
\end{tabular}

Notes. The given values are based on the stellar parameters from Table 2, and observed photometric periods $P$. Given are hydrostatic adjustment timescales $\tau_{\text {hyd }}=\left(G M_{\star} / R_{\star}^{3}\right)^{-1 / 2}$, escape velocities $v_{\text {esc }}=$ $\left(2 G M_{\star} / R_{\star}\right)^{1 / 2}$, critical rotation velocities $v_{\text {crit }}=\left(G M_{\star}\left(1-\Gamma_{\mathrm{e}}\right) / R_{\star}\right)^{1 / 2}$, rotational velocities $v_{\text {rot }}=2 \pi R_{\star} / p$, and specific angular momenta $j=$ $R_{\star} v_{\text {rot }}$. Mass-loss timescales $M_{\star} / \dot{M}$ are given dependent on the wind clumping factor $D$ (cf. Table 2). References for the photometric periods are (1) Morel et al. (1997); (2) Lamontagne \& Moffat (1987); (3) Morel et al. (1999); (4) St.-Louis et al. (1989); (5) Lefèvre et al. (2005).

from different sources throughout the literature. We note that it is not clear whether the observed variability is caused by rotation/CIRs, and that there are significant uncertainties in the periods, as well as the stellar parameters.

According to our estimates in Table 3, the observed periods are typically 1-2 orders of magnitude larger than the hydrostatic adjustment timescales $\tau_{\text {hyd }}$. This supports rotation as possible reason for the variability, as stellar pulsations, such as the expected strange modes (cf. Glatzel \& Kaltschmidt 2002; Glatzel 2008), have timescales $\lesssim \tau_{\text {hyd }}$. The rotational velocities inferred in Table 3 are in agreement with the rotating single star models by Meynet \& Maeder (2003). E.g., the rotating $40 M_{\odot}$ model from Meynet \& Maeder has a rotational surface velocity of $v_{\text {rot }} \sim 35-60 \mathrm{~km} \mathrm{~s}^{-1}$ in the corresponding evolutionary phase. As discussed in Sect. 1.2, these velocities are likely sufficient to cause the observed line effect, although they are significantly lower than the estimated critical velocities.

For the interpretation of the observed rotational velocities we need to elaborate on the complex envelope/wind structure of WR stars, and the ambiguities in the definition of their surface. To this purpose we distinguish between 1) the convective stellar core (or stellar core); 2) the radiative stellar envelope (or stellar envelope); 3) a potential inflated subsurface zone (or inflated zone); 4) the hydrostatic surface radius (or stellar radius); and 5) the stellar photosphere, which may be located within the stellar wind.

The stellar core (1), and stellar envelope (2) clearly belong to the stellar interior, and are the layers which are included in standard evolutionary models. On top of the stellar envelope, an inflated zone (3) may be responsible for an increase of WR star radii by a factor of a few, in accordance with the large radii observed for many Galactic WR stars (cf. Gräfener et al. 2012). Whether this inflation effect occurs in standard evolutionary models or not depends on the treatment of the outer boundary condition, and dynamical effects (Gräfener et al. 2012; Petrovic et al. 2006).

The stellar radius (4), which corresponds to the spectroscopically determined stellar radius $R_{\star}$, is located on top of this envelope in the static layers just below the sonic point of the stellar wind. As WR-type winds are optically thick, the observed stellar photosphere (5) may be located even further out, within the extended stellar wind. We note that the location of the photosphere 
strongly depends on wavelength, and that it is thus difficult to define a photospheric radius in a meaningful way. The spectroscopically determined stellar radius $R_{\star}$, on the other hand, corresponds to the inner boundary of the employed atmosphere models. However, as $R_{\star}$ is not directly observed, it relies on the wind structure adopted within the models. For the interpretation of our results in Table 3 it is thus necessary to estimate the uncertainty in $R_{\star}$.

If we conservatively assume that the uncertainty in $R_{\star}$ is of the same order of magnitude as the wind extension, we can use the relations by de Loore et al. (1982) as an estimate. It turns out that for all objects in Table 3 the estimated wind extension is rather high, with $R_{\mathrm{ph}} \sim 2 \ldots 3 R_{\star}$. There are thus substantial uncertainties in $R_{\star}$. As our observable is the rotation period $P$, the derived rotation parameter $\omega$ becomes

$\omega=\frac{v_{\text {rot }}}{v_{\text {crit }}}=\frac{2 \pi}{P} \sqrt{\frac{R_{\star}^{3}}{G M_{\star}\left(1-\Gamma_{\mathrm{e}}\right)}}$,

and the derived specific angular momentum $j$ at the stellar equator becomes

$j=R_{\star} v_{\mathrm{rot}}=\frac{2 \pi R_{\star}^{2}}{P}$,

based on our definitions of $v_{\text {rot }}$, and $v_{\text {crit }}{ }^{3}$ in Table 3 . The uncertainty in $R_{\star}$ thus translates to a substantial uncertainty of a factor $3 \ldots .5$ in $\omega$, and $4 \ldots 9$ in $j$.

An additional point to consider is that cool WR stars may be subject to the envelope inflation effect. In this case the stellar core (1) and envelope (2) may be hidden below an inflated zone (3), with an extremely low density. The question whether the derived rotational velocities and angular momenta in Table 3 are meaningful, depends on whether the angular momentum in subphotospheric layers (i.e. within the stellar wind, or an inflated stellar envelope) is conserved or not. E.g., in the case of strong angular momentum coupling, the derived surface angular momenta may be over-estimated (in the most extreme case the subphotospheric layers would be rigidly rotating). As the density of these layers is very small, it seems however more likely that the angular momentum coupling is weak. Moreover, as the mass of the inflated layers is very small $\left(<0.01 M_{\odot}\right)$, they are removed by mass-loss, and replaced by material from the stellar envelope (2), on timescales much shorter than the secular timescale. In this case the observed specific angular momenta $j$ should correctly reflect the values on top of the stellar envelope (2), and thus be comparable to the surface values from stellar evolutionary models.

In Sect. 1.2, we already mentioned that the presence of largescale magnetic fields may be an interesting alternative explanation for the detection of photometric variability, and spectropolarimetric signatures. According to Wade (2011), this scenario is however very unlikely, as WR winds would require very strong magnetic fields to produce the observed spectropolarimetric signatures. For WR 6 Wade (2011, and references therein) estimate a required field strength of $\sim 1.6 \mathrm{kG}$, which is significantly higher than their estimated upper limit of $300 \mathrm{G}$ for this star. Moreover, the observed rotational period may be difficult to reconcile with

\footnotetext{
3 To compute $v_{\text {crit }}$ we use the effective stellar mass, corrected for radiation pressure on free electrons $M_{\star}\left(1-\Gamma_{\mathrm{e}}\right)$. As in reality also other opacities contribute to the total Eddington factor $\Gamma$, the effective mass may be smaller, and $v_{\text {crit }}$ lower. The definition based on $\Gamma_{\mathrm{e}}$ thus still poses an upper limit to the rotational velocity $v_{\text {rot }}$. A definition using the total $\Gamma$ is impractical, as $\Gamma$ is a complex function of density and temperature.
}

the short spin-down time resulting from a strong magnetic field $(\sim 0.4 \mathrm{Myr})$.

\subsubsection{An evolutionary channel towards LGRBs?}

Whether stars can explode as LGRBs or not depends on the angular momentum left in the stellar core before the final collapse. The question whether rotating single stars can retain enough angular momentum in their cores throughout the RSG stage, has been addressed by Petrovic et al. (2005). According to this work, the result depends critically on the rotational coupling between the stellar core and envelope, i.e., on the physics of angular momentum transport, potentially involving complex magnetic processes.

For the case of strong coupling (i.e. with magnetic fields), Petrovic et al. found that angular momentum is efficiently transferred from the core to the envelope, leading to a spin-down of the core (i.e. no LGRB), and a spin-up of the surface, already on the main sequence. Their model for a rotating $42 M_{\odot}$ star reaches a very high specific surface angular momentum of $\log \left(j / \frac{\mathrm{cm}^{2}}{\mathrm{~s}}\right) \simeq 20$ at the onset of He-burning.

For the case of weak coupling (i.e. without magnetic fields), Petrovic et al. found that, due to the $\mu$-gradient between H-rich envelope and He-core, rotating single stars maintain a high angular momentum in their cores throughout the RSG phase. As only a small portion of the angular momentum is transferred to the envelope, the specific angular momenta at the surface are much lower than for the magnetic case $\left(\log \left(j / \frac{\mathrm{cm}^{2}}{\mathrm{~s}}\right) \simeq 17.5-19\right)$. These values compare notably well with our estimates from Table 3. Petrovic et al. conclude that such non-magnetic stars may explode as LGRBs, if they end their lives shortly after the RSG phase, avoiding the angular momentum loss during the WR phase.

Hirschi et al. (2005) computed a large number of nonmagnetic LGRB progenitor models for various metallicities. Also in these models the core angular momentum is preserved throughout the RSG phase. As a consequence, they predict too many LGRBs, and demand for additional constraints to explain the low number of LGRBs observed. According to the models by Meynet \& Maeder (2003), which are very similar to the ones by Hirschi et al., the rotational velocities are predicted to increase moderately during later WR phases. This is in contrast to our observation that more evolved WR stars hardly ever show rotational signatures. For example, in the rotating $40 M_{\odot}$ model from Meynet \& Maeder (2003), the WR star spins up from $v_{\text {rot }} \sim 35-60 \mathrm{~km} \mathrm{~s}^{-1}$ in the direct post-RSG phase, to a velocity of $v_{\text {rot }} \sim 90 \mathrm{~km} \mathrm{~s}^{-1}$ before its final collapse. Our results thus seem to suggest that the angular momentum loss in the WR phase is higher than predicted. It is however difficult to draw firm conclusions at this point, because the wind geometry, and thus the spectropolarimetric signal, will depend on a variety of stellar parameters. E.g., in the same model, the critical rotation parameter $\omega=v_{\text {rot }} / v_{\text {crit }}$ is predicted to vary between values of 0.19 and 0.04 throughout the WR phase.

To estimate the timescale of angular momentum loss during the WR phase, we can use the mass-loss timescales $M_{\star} / \dot{M}$ from Table 3 . Dependent on the wind clumping factor $D$, which is found to be of the order of $4 \ldots 16$ for WR stars (Hamann \& Koesterke 1998), the resulting values of $M_{\star} / \dot{M}$ are of the order of $4 \ldots 8 \times 10^{5} \mathrm{yr}$. If the observed specific surface angular momenta $j$ represent a steady state, the angular momentum lost on this timescale should be the order of $j \times M_{\star}$. 
However, as the specific angular momentum is usually increasing towards the stellar surface, the total stellar angular momentum is much smaller than $j \times M_{\star}$ (cf. Langer 1998). Consequently, only a small fraction $f$ of the stellar mass needs to be removed to spin down the star significantly. This lowers the timescale of angular momentum loss to $f \times M_{\star} / \dot{M} \sim$ $4 \ldots 8 \times 10^{4} \mathrm{yr}$, where we have adopted $f \sim 0.1$ (e.g. Packet 1981 ). This value is supported by our detection of enhanced rotational velocities only for WR stars in the nebular phase, which implies that the timescale for angular momentum loss should indeed be smaller than the nebular dissipation timescale $\left(\sim 8 \times 10^{4} \mathrm{yr}\right.$, van Marle et al. 2005).

Following this argument, the average value of $j$ in the stellar interior should be about 10 times smaller than the observed surface value. The presently observed equatorial values of $\log \left(j / \frac{\mathrm{cm}}{\mathrm{s}}\right)=18 \ldots 19$ would then translate to $\log \left(j / \frac{\mathrm{cm}}{\mathrm{s}}\right) \lessgtr 17 \ldots 18$ in the stellar interior. Even in view of the involved uncertainties, this would be near or in excess of the threshold for the formation of a collapsar and LGRB $\left(j \gtrsim 3 \times 10^{16} \mathrm{~cm}^{2} / \mathrm{s}\right.$; MacFadyen $\&$ Woosley 1999), in accordance with the non-magnetic LGRB progenitor models from Petrovic et al. (2005) discussed before.

Although the properties of the objects in Table 3 are in agreement with being LGRB progenitors, we are of course not able to predict on which timescales the individual objects will end their lives, i.e., whether they will finally explode as a LGRB or not. However, they seem to represent a dominant evolutionary channel towards WR stars with enhanced rotation rates in the Galaxy, via a preceding RSG, or LBV phase. This is interesting, as similar stars have been discussed as LGRB progenitors before, to explain the observed absorption features in some LGRB afterglow spectra (cf. Sect. 1.3).

In Sect. 3.1.1 we discussed that rotation is a necessary condition to form the objects in our line-effect sample in a nonnegligible abundance. Dependent on the internal angular momentum coupling in the main-sequence phase, this may be achieved by a single fast rotating star, as for the models by Meynet \& Maeder (2003), or by adding angular momentum, e.g. by mass exchange in a binary system (cf. our discussion in Sect. 3.2). The case of mass exchange on the main-sequence has been investigated by Petrovic et al. (2005). In this scenario the primary transfers mass to the secondary when it starts to fill its Roche-lobe. Consequently the secondary spins up, but the spinup is compensated by the spin-down due to tidal coupling in the binary system. After the former primary explodes as a supernova, the remaining secondary turns out to have very similar properties as the rotating single star case. In both scenarios the non-magnetic case leads to post-RSG/LBV WR stars with high angular momenta in the core.

Notably, the scenarios discussed here differ fundamentally from the scenario by Yoon \& Langer (2005), where the $\mathrm{RSG} / \mathrm{LBV}$ phase is completely avoided. In this scenario magnetic, fast rotating stars evolve chemically homogeneous due to strong rotational mixing. In combination with low WR massloss rates at low metallicities (Vink \& de Koter 2005; Gräfener \& Hamann 2008), they retain their angular momentum and explode as LGRBs (the formation of complex shell geometries around these objects is described by van Marle et al. 2008). This scenario strongly favors low-metallicity environments, in agreement with observed trends in GRB host galaxies (Modjaz et al. 2008, 2011). More recently, LGRBs have however also been detected at high metallicities (Graham et al. 2009; Levesque et al. 2010a,b; Soderberg et al. 2010). The evolutionary channel discussed here may thus play a (relatively) more important role in the high-metallicity regime.

\subsection{Comparison with GRB absorption features}

An additional aspect of our work is to examine whether the putatively CS absorption features, as observed in GRB 020813 and GRB 021004 (cf. Sect. 1.3), can also be found in Galactic WR stars. The observed broad GRB absorption features span a velocity range of $130-630 \mathrm{~km} \mathrm{~s}^{-1}$, in agreement with hydrodynamic models for CS ejecta shells of post-RSG WR stars (van Marle et al. 2005).

According to our results from Sect. 2.3, the C IV absorption features in Galactic WR stars are different from the GRB features. In particular, the velocities detected in C IV turn out to be much lower than those observed in GRB afterglows (up to $\sim 200 \mathrm{~km} \mathrm{~s}^{-1}$ ). In addition, we only find weak evidence in Sect. 2.4 that the CS velocities of WR stars with a line-effect are enhanced with respect to others. The CS properties of typical LGRB progenitors may thus either differ from Galactic WR stars, or the WR/GRB absorption features are not due to CS material. E.g., St.-Louis \& Smith (1991) suggested that highvelocity absorptions in $\mathrm{Cyg}$ OB1 \& OB3 are formed in a supershell surrounding Cyg OB1. On the other hand, differences in the CS properties around GRBs may indeed be expected, as the strength, and the velocity of the WR winds, which are responsible for shaping the ejecta shell, depend on metallicity (Vink \& de Koter 2005; Gräfener \& Hamann 2008). Moreover, due to the clumpiness of the CS material, the detection probability of the CS absorption features is expected to lie in the range of 20-60\% (van Marle et al. 2005), i.e. many CS features may actually be missed by our method.

One of the best studied nebulae in our sample is the ejecta nebula RCW 58 around WR 40. The morphology and radius of this nebula $\left(r \sim 2.7 \mathrm{pc}^{4}\right)$ are strikingly similar to the model predictions by van Marle et al. (2005). It thus appears very likely, that it is a post-RSG, or post-LBV ejecta shell in the breakup phase. Smith et al. (1984) found UV absorption features in the velocity range of $100-150 \mathrm{~km} \mathrm{~s}^{-1}$ in the spectrum of WR 40 . Moreover, they found a correlation between the measured velocities for different ions, and their ionization potential. They concluded that the observed high-velocity features are due to shocks within the structured nebula. For the visible nebula itself, Smith et al. (1988) measured slightly lower velocities from nebular emission lines, in agreement with a spherically expanding shell with $v \sim 90 \mathrm{~km} \mathrm{~s}^{-1}$. Notably, this value is much lower than the prediction of van Marle et al. (2005).

Nichols-Bohlin \& Fesen (1986, 1990, 1993), on the other hand, found similar high-velocity features also on sight lines far beyond the ejecta nebulae of WR 6, WR 40, and WR 136. They concluded that the high-velocity features are not correlated with the nebula itself, but with much larger structures, which are also associated with the WR stars. For example, for WR 40 they identified a structure in IRAS data, with a size of $\sim 100 \mathrm{pc} \times 150 \mathrm{pc}$, nearly centered on WR 40. Nichols \& Fesen (1994) interpreted these structures as evolved supernova remnants within a binary scenario with mass exchange, similar to the one by Petrovic et al. (2005). According to these works, at least three of the six stars in our line-effect sample (WR 6, WR 40, and WR 136) would thus display a double shell structure that may result from binary evolution. An inner ejecta nebula with enhanced velocities $(r \sim 3 \mathrm{pc}$, $v \sim 90 \mathrm{~km} \mathrm{~s}^{-1}$ for WR 40), and a much larger structure with even higher velocities, $\left(r \sim 100 \mathrm{pc}, v \sim 150 \mathrm{~km} \mathrm{~s}^{-1}\right.$ for WR 40) that may result from a supernova explosion of the former primary.

\footnotetext{
${ }^{4}$ Based on a photometric distance estimate of $2.3 \mathrm{kpc}$ from van der Hucht (2001).
} 
Most notably, the large size of the outer high-velocity shells as observed for WR 6, WR 40, and WR 136 may resolve the main problem with the interpretation of GRB absorption features as CS material. If the absorptions would originate from typical ejecta nebulae with a size of only a few pc, one would expect a strong time-dependence due to the ionizing radiation from the GRB itself. According to Lazzati (2005) the absence of a strong time evolution in these features constrains the distance between the absorber and the GRB to a minimum of several tens of parsecs. The large high-velocity shells around WR 6, WR 40, and WR 136, which are presently detectable as high-velocity absorptions in the UV, would thus remain detectable as stationary absorptions if these stars explode as LGRBs.

\section{Conclusions}

In this work we examined the circumstellar, and spectropolarimetric properties of Galactic WR stars. We used the spectropolarimetric line effect as an indicator of enhanced rotation, and the presence of "possible ejecta nebulae" as an indicator of CS ejecta from a recent RSG or LBV phase.

Based on these two assumptions we conclude that the group of $\sim 23 \%$ of Galactic WR stars with CS ejecta have enhanced rotation parameters $\omega=v_{\text {rot }} / v_{\text {crit }}$ compared to the majority of WR stars. This confirms the strong correlation between rotation and CS ejecta from Vink et al. (2011a). The largest part of rotating WR stars in the Galaxy are thus likely "young" WR stars, shortly after the RSG/LBV stage. This implies that the rotational coupling between core and envelope in the RSG/LBV phase is small enough to maintain substantial angular momentum at the beginning of the WR phase. Such objects have previously been discussed as potential LGRB progenitors if they explode early enough after the RSG/LBV phase (Petrovic et al. 2005).

In fact we estimate rotation parameters in the range $\omega=$ 0.04...0.25 from photometric periods, corresponding to moderate rotation speeds of $36 \ldots 120 \mathrm{~km} \mathrm{~s}^{-1}$. These values are very uncertain, but are in agreement with predicted specific surface angular momenta for LGRB progenitors, e.g. from Petrovic et al. (2005). A subgroup of stars exploding in this early WR stage may thus indeed represent an evolutionary channel towards LGRBs at high metallicities, comparable to our Galaxy.

Furthermore, we find a very small incidence of WR stars with signatures of enhanced rotation in later evolutionary phases. This implies that angular momentum is efficiently lost due to the strong mass loss in the WR phase. According to our results, the angular momentum loss in the WR phase appears to be larger than predicted, however, firm conclusions would require a more detailed knowledge of the influence of rotation on WR-type mass loss.

"Young" WR stars after a RSG/LBV phase have previously been discussed as LGRB progenitors to explain the circumstellar absorption features as observed in some LGRB afterglows (GRB 020813 and GRB 021004). Here we identified similar absorption features in the UV spectra of some putatively rotating WR stars, albeit with much lower velocities. According to previous works, these features are most likely associated with extended CS structures $(r \sim 100 \mathrm{pc})$, which may be evolved supernova remnants from a former binary partner (Nichols \& Fesen 1994). These structures are large enough to explain the stability of the observed GRB absorptions against the ionizing radiation from the GRB itself, which may resolve a fundamental problem with their interpretation as CS material. Moreover, if these enigmatic structures were supernova remnants, this may indicate a binary scenario with mass transfer as a channel towards LGRB formation.
We only find one evolved WC star in our sample with signatures of enhanced rotation. This star has previously been discussed as a possible LGRB progenitor by Chené \& St.-Louis (2010), and may represent an evolutionary channel via chemically homogeneous evolution, such as the magnetic single star, or binary models by Yoon \& Langer (2005); Cantiello et al. (2007). At Galactic metallicity, such stars are however not expected to explode as LGRBs. The rotating post RSG/LBV stars detected in our present work may thus represent an evolutionary channel towards LGRBs that is particularly important at high metallicities.

Acknowledgements. We thank the anonymous referee for his comments which helped clarify the paper substantially. G.G. and J.S.V. thank STFC for financial support under grant No. ST/J001082/1.

\section{References}

Berdyugin, A. V., \& Harries, T. J. 1999, A\&A, 352, 177 Bjorkman, J. E., \& Cassinelli, J. P. 1993, ApJ, 409, 429

Brown, J. C., McLean, I. S., \& Emslie, A. G. 1978, A\&A, 68, 415

Cantiello, M., Yoon, S.-C., Langer, N., \& Livio, M. 2007, A\&A, 465, L29

Chené, A.-N., \& St.-Louis, N. 2010, ApJ, 716, 929

Chu, Y.-H. 1981, ApJ, 249, 195

Chu, Y. H. 1991, in Wolf-Rayet Stars and Interrelations with Other Massive Stars in Galaxies, eds. K. A. van der Hucht, \& B. Hidayat, IAU Symp., 143, 349 Cranmer, S. R., \& Owocki, S. P. 1996, ApJ, 462, 469

de Loore, C., Hellings, P., \& Lamers, H. J. G. 1982, in Wolf-Rayet Stars: Observations, Physics, Evolution, eds. C. W. H. De Loore, \& A. J. Willis, IAU Symp., 99, 53

Dessart, L., \& Owocki, S. P. 2002, A\&A, 383, 1113

Dessart, L., Crowther, P. A., Hillier, D. J., et al. 2000, MNRAS, 315, 407

Fiore, F., D'Elia, V., Lazzati, D., et al. 2005, ApJ, 624, 853

Flores, A., Koenigsberger, G., Cardona, O., \& de la Cruz, L. 2007, AJ, 133, 2859

Fox, A. J., Ledoux, C., Vreeswijk, P. M., Smette, A., \& Jaunsen, A. O. 2008, A\&A, 491, 189

Galama, T. J., Vreeswijk, P. M., van Paradijs, J., et al. 1998, Nature, 395, 670

Glatzel, W. 2008, in Hydrogen-deficient stars, eds. A. Werner, \& T. Rauch (San Francisco: ASP), ASP Conf. Ser., 391, 307

Glatzel, W., \& Kaltschmidt, H. O. 2002, MNRAS, 337, 743

Gräfener, G., \& Hamann, W.-R. 2008, A\&A, 482, 945

Gräfener, G., Vink, J. S., de Koter, A., \& Langer, N. 2011, A\&A, 535, A56

Gräfener, G., Owocki, S. P., \& Vink, J. S. 2012, A\&A, 538, A40

Graham, J. F., Fruchter, A. S., Kewley, L. J., et al. 2009, in AIP Conf. Ser. 1133, eds. C. Meegan, C. Kouveliotou, \& N. Gehrels, 269

Hamann, W.-R., \& Koesterke, L. 1998, A\&A, 335, 1003

Hamann, W.-R., Gräfener, G., \& Liermann, A. 2006, A\&A, 457, 1015

Harries, T. J., \& Hilditch, R. W. 1997, MNRAS, 291, 544

Harries, T. J., \& Howarth, I. D. 1996, A\&A, 310, 533

Harries, T. J., Hillier, D. J., \& Howarth, I. D. 1998, MNRAS, 296, 1072

Harries, T. J., Howarth, I. D., Schulte-Ladbeck, R. E., \& Hillier, D. J. 1999, MNRAS, 302, 499

Herald, J. E., Hillier, D. J., \& Schulte-Ladbeck, R. E. 2001, ApJ, 548, 932

Hirschi, R., Meynet, G., \& Maeder, A. 2005, A\&A, 443, 581

Hjorth, J., Sollerman, J., Møller, P., et al. 2003, Nature, 423, 847

Ignace, R., Cassinelli, J. P., \& Bjorkman, J. E. 1996, ApJ, 459, 671

Lamontagne, R., \& Moffat, A. F. J. 1987, AJ, 94, 1008

Langer, N. 1998, A\&A, 329, 551

Langer, N., Hamann, W.-R., Lennon, M., et al. 1994, A\&A, 290, 819

Lazzati, D. 2005, Nuovo Cimento C Geophysics Space Physics C, 28, 575

Lefèvre, L., Marchenko, S. V., Lépine, S., et al. 2005, MNRAS, 360, 141

Levesque, E. M., Kewley, L. J., Graham, J. F., \& Fruchter, A. S. 2010a, ApJ, 712, L26

Levesque, E. M., Soderberg, A. M., Foley, R. J., et al. 2010b, ApJ, 709, L26

MacFadyen, A. I., \& Woosley, S. E. 1999, ApJ, 524, 262

Malesani, D., Tagliaferri, G., Chincarini, G., et al. 2004, ApJ, 609, L5

Marston, A. P. 1997, ApJ, 475, 188

Marston, A. P., Chu, Y.-H., \& Garcia-Segura, G. 1994a, ApJS, 93, 229

Marston, A. P., Yocum, D. R., Garcia-Segura, G., \& Chu, Y.-H. 1994b, ApJS, 95, 151

Meynet, G., \& Maeder, A. 2003, A\&A, 404, 975

Meynet, G., \& Maeder, A. 2005, A\&A, 429, 581

Miller, G. J., \& Chu, Y.-H. 1993, ApJS, 85, 137

Mirabal, N., Halpern, J. P., Chornock, R., et al. 2003, ApJ, 595, 935 
Modjaz, M., Kewley, L., Kirshner, R. P., et al. 2008, AJ, 135, 1136 Modjaz, M., Kewley, L., Bloom, J. S., et al. 2011, ApJ, 731, L4

Morel, T., St.-Louis, N., \& Marchenko, S. V. 1997, ApJ, 482, 470

Morel, T., Marchenko, S. V., Eenens, P. R. J., et al. 1999, ApJ, 518, 428

Nichols, J. S., \& Fesen, R. A. 1994, A\&A, 291, 283

Nichols-Bohlin, J., \& Fesen, R. A. 1986, AJ, 92, 642

Nichols-Bohlin, J., \& Fesen, R. A. 1990, ApJ, 353, 281

Nichols-Bohlin, J., \& Fesen, R. A. 1993, AJ, 105, 672

Owocki, S. P., \& Gayley, K. G. 1997, in Luminous Blue Variables: Massive Stars in Transition, eds. A. Nota, \& H. Lamers, ASP Conf. Ser., 120, 121

Packet, W. 1981, A\&A, 102, 17

Pelupessy, I., Lamers, H. J. G. L. M., \& Vink, J. S. 2000, A\&A, 359, 695

Petrovic, J., Langer, N., Yoon, S.-C., \& Heger, A. 2005, A\&A, 435, 247

Petrovic, J., Pols, O., \& Langer, N. 2006, A\&A, 450, 219

Poeckert, R., \& Marlborough, J. M. 1977, ApJ, 218, 220

Schaefer, B. E., Gerardy, C. L., Höflich, P., et al. 2003, ApJ, 588, 387

Schmidt, G. D. 1988, Spectropolarimetry as a probe of the structure of

Wolf-Rayet envelopes, eds. G. V. Coyne, A. M. Magalhaes, A. F. Moffat,

R. E. Schulte-Ladbeck, \& S. Tapia, 641

Schulte-Ladbeck, R. E. 1994, Ap\&SS, 221, 347

Schulte-Ladbeck, R. E., Clayton, G. C., Hillier, D. J., Harries, T. J., \& Howarth, I. D. 1994, ApJ, 429, 846

Smith, L. J., Willis, A. J., \& Wilson, R. 1980, MNRAS, 191, 339

Smith, L. J., Pettini, M., Dyson, J. E., \& Hartquist, T. W. 1984, MNRAS, 211 , 679

Smith, L. J., Lloyd, C., \& Walker, E. N. 1985, A\&A, 146, 307
Smith, L. J., Pettini, M., Dyson, J. E., \& Hartquist, T. W. 1988, MNRAS, 234, 625

Soderberg, A. M., Chakraborti, S., Pignata, G., et al. 2010, Nature, 463, 513

St.-Louis, N., \& Smith, L. J. 1991, A\&A, 252, 781

St.-Louis, N., Smith, L. J., Stevens, I. R., et al. 1989, A\&A, 226, 249

St.-Louis, N., Moffat, A. F. J., Lapointe, L., et al. 1993, ApJ, 410, 342

Starling, R. L. C., Wijers, R. A. M. J., Hughes, M. A., et al. 2005, MNRAS, 360, 305

Stock, D. J., \& Barlow, M. J. 2010, MNRAS, 409, 1429

van der Hucht, K. A. 2001, New Astron. Rev., 45, 135

van Marle, A. J., Langer, N., \& García-Segura, G. 2005, A\&A, 444, 837

van Marle, A. J., Langer, N., Yoon, S.-C., \& García-Segura, G. 2008, A\&A, 478, 769

Vink, J. S. 2007, A\&A, 469, 707

Vink, J. S., \& de Koter, A. 2005, A\&A, 442, 587

Vink, J. S., Davies, B., Harries, T. J., Oudmaijer, R. D., \& Walborn, N. R. 2009, A\&A, 505, 743

Vink, J. S., Gräfener, G., \& Harries, T. J. 2011a, A\&A, 536, L10

Vink, J. S., Muijres, L. E., Anthonisse, B., et al. 2011b, A\&A, 531, A132

Wade, G. A. 2011 [arXiv: 1111.0212]

Whitney, B. A., Schulte-Ladbeck, R. E., Aspin, C., et al. 1988, in BAAS, 20, 1013

Woosley, S. E. 1993, ApJ, 405, 273

Woosley, S. E., Langer, N., \& Weaver, T. A. 1993, ApJ, 411, 823

Yoon, S.-C., \& Langer, N. 2005, A\&A, 443, 643

Zeh, A., Klose, S., \& Hartmann, D. H. 2004, ApJ, 609, 952 
Table 4. WR stars with IUE high-resolution data: circumstellar, and spectropolarimetric properties.

\begin{tabular}{|c|c|c|c|c|c|c|c|c|c|}
\hline WR & HD & IUE No. & Sp. type & LE & $\mathrm{RN}$ & $\mathrm{NF}$ & $\mathrm{C}_{\text {IV }} 1548$ & $\mathrm{C}_{\text {IV }} 1550$ & \\
\hline 006 & 50896 & SWP04065 & WN4 & + & $\mathrm{W}, \mathrm{E}$ & $\mathrm{NF}++$ & 93.7 & 75.4 & sing \\
\hline 008 & 62910 & SWP27473 & WN7/WCE+? & & $\mathrm{E}$ & NF- & 70.8 & 80.0 & split \\
\hline 010 & 65865 & SWP29703 & WN5ha $(+\mathrm{A} 2 \mathrm{~V})$ & & - & & - & - & noise \\
\hline 011 & 68273 & SWP46918 & $\mathrm{WC} 8+07.5 \mathrm{III}-\mathrm{V}$ & & $\mathrm{E}$ & $\mathrm{NF}-$ & 38.7 & 34.4 & sing \\
\hline 014 & 76536 & SWP10105 & WC7+? & - & $\mathrm{R}$ & $\mathrm{NF}-$ & 86.8 & 48.0 & sing/mult \\
\hline 016 & 86161 & SWP13893 & WN8h & + & W/E & $\mathrm{NF}-$ & $<300$ & 278.8 & forest \\
\hline 022 & 92740 & SWP04067 & WN7h+O9III-V & & W? & $\mathrm{NF}+$ & 153.4 & 150.8 & forest \\
\hline 023 & 92809 & SWP11281 & WC6 & & $\mathrm{R}, \mathrm{W}$ & $\mathrm{NF}+$ & 91.4 & 71.0 & sing/mult \\
\hline 024 & 93131 & SWP04332 & WN6ha & - & - & $\mathrm{NF}+$ & 125.9 & 107.5 & mult/forest \\
\hline 025 & 93162 & SWP13993 & $\mathrm{WN} 6 \mathrm{~h}+\mathrm{O} 4 \mathrm{f}$ & & - & $\mathrm{NF}+$ & 118.9 & 109.6 & mult/forest \\
\hline 040 & 96548 & SWP27322 & WN8h & + & $\mathrm{W} / \mathrm{E}$ & $\mathrm{NF}++$ & 281.5 & 292.7 & forest \\
\hline 042 & 97152 & SWP16085 & $\mathrm{WC} 7+\mathrm{O} 7 \mathrm{~V}$ & & $\mathrm{E}$ & $\mathrm{NF}+$ & 187.7 & 194.4 & mult/forest \\
\hline 046 & 104994 & SWP41778 & $\mathrm{WN} 3 \mathrm{p}+\mathrm{OB} ?$ & & - & $\mathrm{NF}-$ & 50.3 & 52.6 & single \\
\hline 048 & 113904 & SWP05852 & WC6 (+O9.5/B0Iab) & & $\mathrm{R}$ & NF- & 82.3 & 91.4 & single \\
\hline 052 & 115473 & SWP15105 & WC4 & & $\mathrm{R}, \mathrm{E} / \mathrm{R}$ & NF- & 61.8 & 70.8 & broad \\
\hline 057 & 119078 & SWP16581 & WC8 & & $\mathrm{E}$ ? & $\mathrm{NF}-$ & 80.0 & 79.8 & mult \\
\hline 069 & 136488 & SWP13816 & WC9d+OB & & - & $\mathrm{NF}-$ & - & - & noise \\
\hline 071 & 143414 & SWP16976 & WN6+OB? & & $\mathrm{E}$ & $\mathrm{NF}++$ & 102.8 & 118.7 & split \\
\hline 078 & 151932 & SWP33475 & WN7h & - & - & $\mathrm{NF}-$ & 87.0 & 59.4 & sing \\
\hline 079 & 152270 & SWP15129 & $\mathrm{WC7}+\mathrm{O} 5-8$ & & - & $\mathrm{NF}+$ & 89.3 & 96.1 & sing/mult \\
\hline 085 & $155603 \mathrm{~B}$ & SWP45275 & WN6h+OB? & & $\mathrm{W} / \mathrm{E}$ & & - & - & noise \\
\hline 090 & 156385 & SWP02891 & WC7 & & - & NF- & 48.0 & 43.5 & $\operatorname{sing}$ \\
\hline 092 & 157451 & SWP16835 & WC9 & & - & NF- & - & 139.4 & forest \\
\hline 103 & 164270 & SWP08156 & WC9d+? & & - & $\mathrm{NF}-$ & - & 61.7 & sing \\
\hline 111 & 165763 & SWP02872 & WC5 & - & - & $\mathrm{NF}-$ & 91.4 & - & sing/mult \\
\hline 113 & 168206 & SWP34037 & WC8d+08-9IV & & RN? & & - & - & noise \\
\hline 128 & 187282 & SWP06999 & $\mathrm{WN} 4(\mathrm{~h})+\mathrm{OB} ?$ & - & $\mathrm{R}$ & $\mathrm{NF}-$ & 66.4 & 52.6 & single \\
\hline 133 & 190918 & SWP14715 & WN5+O9I & - & RN?? & $\mathrm{NF}+$ & 114.5 & 105.2 & mult/forest \\
\hline 134 & 191765 & SWP04088 & WN6 & + & W & $\mathrm{NF}+$ & 114.5 & 112.0 & mult/forest \\
\hline 135 & 192103 & SWP04086 & WC8 & - & - & $\mathrm{NF}+$ & 98.4 & 98.2 & mult/forest \\
\hline 136 & 192163 & SWP08812 & WN6(h) & + & $\mathrm{W} / \mathrm{E}$ & $\mathrm{NF}+$ & 151.0 & 139.4 & mult/split \\
\hline 137 & 192641 & SWP25628 & $\mathrm{WC} 7 \mathrm{pd}+\mathrm{O} 9$ & + & - & $\mathrm{NF}+$ & 112.0 & 109.6 & mult/forest \\
\hline 138 & 193077 & SWP07000 & WN5+B? & - & - & $\mathrm{NF}+$ & 103.0 & 93.8 & mult/split \\
\hline 139 & 193576 & SWP25990 & WN5+O6III-V & * & - & $\mathrm{NF}-$ & 66.2 & 59.4 & sing/mult \\
\hline 140 & 193793 & SWP08004 & WC7pd+O4-5 & & - & $\mathrm{NF}-$ & 77.7 & 64.0 & sing \\
\hline 148 & 197406 & - & $\mathrm{WN} 8 \mathrm{~h}+\mathrm{B} 3 \mathrm{IV} / \mathrm{BH}$ & - & - & $\mathrm{NF}++$ & - & - & - \\
\hline $153 \mathrm{ab}$ & 211853 & SWP15100 & WN6/WCE+O6I & - & RN?? & $\mathrm{NF}-$ & 93.7 & 79.9 & mult \\
\hline 155 & 214419 & SWP38134 & WN6+O9II-Ib & $*$ & - & $\mathrm{NF}-$ & 103.1 & 130.1 & mult/forest \\
\hline
\end{tabular}

Notes. WR designations by van der Hucht (2001); HD numbers; IUE numbers; Spectral types from van der Hucht (2001); LE, RN, NF, C IV same as in Table 1. The last column gives a tentative classification of the morphology of the observed C IV absorption features (cf. Fig. 1). 
G. Gräfener et al.: Rotating Wolf-Rayet stars in a post RSG/LBV phase

Table 5. Ring nebulae around 172 Galactic WR stars, from the Surveys by Marston (1997), and Miller \& Chu (1993).

\begin{tabular}{|c|c|c|c|c|c|c|c|c|c|c|c|c|c|c|}
\hline WR & Ma97 & MC93 & Ch91 & SB10 & WR & Ma97 & МC93 & Ch91 & SB10 & WR & Ma97 & MC93 & Ch91 & SB10 \\
\hline 1 & & - & & & 44 & - & & & & 103 & - & & & \\
\hline 2 & & - & & & $44 \mathrm{a}$ & - & & & & 104 & & - & & \\
\hline 3 & & - & & & 45 & - & & & & 105 & - & - & & \\
\hline 4 & & - & & & 46 & - & & & & 106 & & - & & \\
\hline 5 & & - & & & 47 & - & & & & 107 & & - & & \\
\hline 6 & E & $\mathrm{RN}$ & W & $\mathrm{E}$ & 48 & - & & $\mathrm{R}$ & & 108 & & - & & \\
\hline 7 & $\mathrm{~W} / \mathrm{E}$ & RN & W & W/E & $48 a$ & - & & & & 109 & - & - & & \\
\hline 8 & - & & & E & 49 & - & & & & 110 & & - & & \\
\hline 9 & - & & & & 50 & - & & & & 111 & & - & & \\
\hline 10 & - & & & & 51 & - & & & & 112 & & - & & \\
\hline 11 & E & & & & 52 & $\mathrm{E} / \mathrm{R}$ & & $\mathrm{R}$ & & 113 & & RN? & & \\
\hline 12 & - & & & & 53 & - & & & & 114 & & - & & \\
\hline 13 & - & & & & 54 & $\mathrm{E} / \mathrm{R}$ & & & & 115 & & - & & \\
\hline 14 & $\mathrm{R}$ & & & & 55 & $\mathrm{R}$ & & $\mathrm{R}$ & & 116 & & RN? & & E \\
\hline 15 & - & & & & 56 & - & & & & 117 & & - & & \\
\hline 16 & $\mathrm{~W} / \mathrm{E}$ & & & $\mathrm{W} / \mathrm{E}$ & 57 & E? & & & & 118 & & - & & \\
\hline 17 & - & & & & 58 & - & & & & 119 & & - & & \\
\hline 18 & $\mathrm{~W} / \mathrm{E}$ & & W & W/E? & 59 & - & & & & 120 & & - & & \\
\hline 19 & - & & & & 60 & E? & & & & 121 & & - & & \\
\hline $19 a$ & - & & & & 61 & - & & & & 122 & & - & & \\
\hline 20 & $\mathrm{R}$ & & & & 62 & - & & & & 123 & & - & & \\
\hline $20 a$ & W & & & & 63 & - & & & & 124 & & $\mathrm{RN}$ & E & E \\
\hline $20 \mathrm{~b}$ & W & & & & 64 & - & & & & 125 & & - & & \\
\hline 21 & - & & & & 65 & W & & & & 126 & & - & & \\
\hline 22 & W? & & & & 66 & - & & & & 127 & & - & & \\
\hline 23 & $\mathrm{R}$ & & W & & 68 & $\mathrm{~W} / \mathrm{E}$ & & & & 128 & & RN & $\mathrm{R}$ & \\
\hline 24 & - & & & & 69 & - & & & & 129 & & - & & \\
\hline 25 & - & & & & 70 & - & & & & 130 & & - & & \\
\hline 26 & - & & & & 71 & E & & & E & 131 & & $\mathrm{RN}$ & $\mathrm{R}$ & \\
\hline 27 & - & & & & 73 & - & & & & 132 & & RN? & & \\
\hline 28 & - & & & & 74 & - & & & & 133 & & RN?? & & \\
\hline 29 & - & & & & 75 & W & & W/E & $\mathrm{W} / \mathrm{E}$ & 134 & & RN & W & \\
\hline 30 & $\mathrm{~W} / \mathrm{E}$ & & & & 76 & - & & & & 135 & & - & & \\
\hline $30 \mathrm{a}$ & - & & & & 77 & W & & & & 136 & & RN & $\mathrm{W} / \mathrm{E}$ & W/E \\
\hline 31 & E & & & & 78 & - & & & & 137 & & - & & \\
\hline $31 \mathrm{c}$ & - & & & & 79 & - & & & & 138 & & - & & \\
\hline 32 & $\mathrm{R}$ & & & & 80 & - & & & & 139 & & - & & \\
\hline 33 & - & & & & 81 & - & & & & 140 & & - & & \\
\hline 34 & - & & & & 82 & - & & & & 141 & & - & & \\
\hline 35 & $\mathrm{R}$ & & & & 83 & - & & & & 142 & & - & & \\
\hline $35 a$ & $\mathrm{R}$ & & & & 84 & - & & & & 143 & & - & & \\
\hline $35 b$ & - & & & & 85 & W/E & & & & 144 & & - & & \\
\hline 36 & $\mathrm{R}$ & & & & 86 & - & & & & 145 & & - & & \\
\hline 37 & - & & & & 87 & W? & & & & 146 & & - & & \\
\hline 38 & E? & & & & 88 & - & & & & 147 & & - & & \\
\hline $38 \mathrm{a}$ & - & & & & 89 & - & & & & 148 & & - & & \\
\hline $38 b$ & - & & & & 90 & - & & & & 149 & & - & & \\
\hline 39 & - & & & & 91 & W/E & & & $\mathrm{R} / \mathrm{E}$ ? & 150 & & - & & \\
\hline 40 & $\mathrm{~W} / \mathrm{E}$ & & $\mathrm{W} / \mathrm{E}$ & $\mathrm{W} / \mathrm{E}$ & 92 & - & & & & 151 & & - & & \\
\hline 41 & - & & & & 93 & W & & & & 152 & & - & & \\
\hline 42 & E & & & & 94 & E & & & & $153 \mathrm{ab}$ & & RN?? & & \\
\hline $42 a$ & - & & & & 95 & - & & & & 154 & & - & & \\
\hline $42 b$ & - & & & & 96 & - & & & & 155 & & - & & \\
\hline $42 c$ & - & & & & 97 & - & & & & 156 & & - & & \\
\hline $42 d$ & $\mathrm{~W} / \mathrm{E}$ & & & & 98 & W/E & & & & 157 & & - & & \\
\hline $43 a$ & - & & & & 100 & - & & & & 158 & & - & & \\
\hline $43 b$ & - & & & & 101 & W/E & & & & & & & & \\
\hline $43 c$ & - & & & & 102 & W & $\mathrm{RN}$ & W & $\mathrm{R} / \mathrm{E}$ & & & & & \\
\hline
\end{tabular}

Notes. WR designations by van der Hucht (2001); nebular classifications by Ma97 (Marston 1997), MC93 (Miller \& Chu 1993), Ch91 (Chu 1991), and SB10 (Stock \& Barlow 2010). Classifications according to Chu (1981): radiatively excited H II regions (R), wind-blown bubbles (W), stellar ejecta (E), no ring nebula (-). Detections by Miller \& Chu (1993): ring nebula (RN), probable ring nebula (RN?), possible ring nebula (RN??), no ring nebula (-). Some stellar designations have been updated with respect to the original works: WR 31a $\rightarrow$ WR 31c; WR 43 is a cluster of WR stars without ring nebula (NGC 3603) $\rightarrow$ WR 42a, WR 42b, WR 42c; WR153 $\rightarrow$ WR 153ab. 